\title{
Multimorbidity predicts quality of life but not motor severity in early Parkinson's disease
}

R Gravell', G W Duncan',3, T K Khoo', D J Burn'1, A A Sayer ${ }^{3,5,6}$, R.A. Barker7, R A Lawson ${ }^{3,5}$, A J Yarnall $3,5,6$

1 Faculty of Medical Science, Newcastle University; 2 Centre for Clinical Brain Sciences, University of Edinburgh; 3 Institute of Neuroscience, Newcastle University; 4 School of Medicine \& Menzies Health Institute Queensland, Griffith University, Australia; 5 Newcastle Institute for Ageing. Newcastle University; 6 AGE Group, Newcastle University; 7 Department of Clinical Neurosciences, Cambridge Centre for Brain Repair

\section{Corresponding Author:}

Rhys Gravell

Faculty of Medicine

Newcastle University

Newcastle upon Tyne

NE4 5PL

rhys-gravell@hotmail.com

Abstract Word Count: 98

Word Count (Excluding abstract): 1499

Number of tables: 2

Number of references: 13

Keywords: Multimorbidity, Parkinson's disease, Quality of life, Motor severity 


\section{Abstract}

Recent guidance by the National Institute for Health and Care Excellence (NICE) focuses on the management of people with multimorbidity, including Parkinson's disease (PD). To date there has been little exploration of this in neurodegenerative diseases. This study aimed to explore the associations between multimorbidity, motor severity and quality of life (QoL) in early PD. Regression analyses determined whether multimorbidity was significantly associated with disease severity and QoL. Multimorbidity was a small but significant predictor of QoL in people with incident PD, but not motor severity, suggesting that they may benefit from a tailored multidisciplinary approach to care. 


\section{Introduction}

Parkinson's disease (PD) is characterised by both motor and non-motor symptoms (NMS) [1]. It is primarily a disease of older people; thus, many will have comorbid disease at the time of diagnosis. Multimorbidity is an evolving concept of particular relevance in later life [2] and to date there has been little study of this in PD. Multimorbidity in PD negatively impacts on many aspects of the disease, including quality of life (QoL) [1]. Multimorbidity is defined as having two or more concurrent chronic physical or mental conditions, and affects $75 \%$ of those over 70 years $[3,4]$. Risk factors include increasing age, low education, and alcohol intake [5]. Multimorbidity has a deleterious and dose-response effect on cognitive function, particularly in those at risk of cognitive impairment [6]. It is associated with increased utilisation of healthcare resources, functional disabilities and hospitalisation $[5,7]$.

Secondary care is often specific with conditions being dealt with in isolation leading to fragmented care [6], therefore the National Institute for Health and Care Excellence (NICE) have produced guidelines on multimorbidity, identifying those that may benefit from multidisciplinary input [4]. These include those with two or more chronic physical or mental health conditions or symptom complexes. Parkinson's disease encompasses symptom complexes such as frailty and pain, therefore is a key target disease from the NICE guidelines. McLean et al. [8] recently highlighted the need for targeted research into multimorbidity in older people.

The aim of this study was to explore the associations between multimorbidity, severity of motor symptoms and QoL in people with early PD. We hypothesized that those with greater multimorbidity scores would have poorer QoL and psychological health. 


\section{Methods}

This was conducted as part of the Incidence of Cognitive Impairment in Cohorts with Longitudinal Evaluation - Parkinson's disease (ICICLE-PD) study, a longitudinal observational study conducted over two sites in Newcastle-upon-Tyne and Cambridgeshire. Participants with newly diagnosed idiopathic PD ( $n=154)$, plus ageand sex- matched unrelated healthy controls $(n=99)$ were recruited from community sources, with the initial aim of comparing multimorbidity in PD with controls [9]. Neurological assessment consisted of a standardised examination. Motor severity was rated with the MDS Unified Parkinson's Disease Rating Scale (MDS-UPDRS-III), and Hoehn and Yahr scale. A global measure of health-related QoL was assessed using the Parkinson's disease Questionnaire (PDQ-39), and depression with the Geriatric Depression Score (GDS-15). Global cognition was assessed using Montreal Cognitive Assessment score (MoCA). Levodopa equivalent daily dose (LEDD) was calculated using Thompson et al.'s formula [10]. Non-motor symptoms were determined using the NMS Questionnaire (NMSQuest). Multimorbidity criteria were applied to the full medication list and past medical history. The presence of multimorbidity was defined in four different ways:

1. A binary classification of multimorbidity based on the definition given in the NICE guidelines [4].

2. Disease count using International Classification of Primary Care-2 (ICPC-2) conditions.

3. Medication counts of all prescribed and non-PD medications.

4. Age-adjusted Charlson Comorbidity Index (CCI). 
Using IBM SPSS V24, data were examined for normality of distribution using Kolmogorov-Smirnov's test and visual histograms. Mann-Whitney $\mathrm{U}$ - and t-tests were performed as appropriate. Pearson's Chi squared tests were used to compare categorical data. Spearman's rank correlations were used to assess correlation. Hierarchical linear regression analysis was conducted to identify predictors of MDSUPDRS-III and PDQ-39 scores. After removal of non-significant predictors, multimorbidity measures, as described above, were individually added to the basic models to determine whether they were significant independent predictors of motor disease and QoL. Given the exploratory nature of the study, no adjustments for multiple testing was made.

\section{Results}

Participants with PD ( $n=154$, mean disease duration 6.0 \pm 4.6 months) and controls $(n=99)$ were well-matched for age, gender and years of education (Supplementary Data). Participants with PD demonstrated a higher level of global cognitive impairment as measured by MoCA, in addition to greater depression scores. The mean number of NMS $(8.4 \pm 4.4$ vs $2.9 \pm 2.6, t=-10.0, p<0.001)$ and mean number of all prescribed medications $(4.9 \pm 3.1$ vs $3.1 \pm 2.8, t=-4.8, p<0.001)$ were significantly greater in $P D$ patients.

The burden of multimorbidity did not differ between PD and control participants as measured by the mean ICPC- 2 conditions ( $2.3 \pm 1.8$ vs. $2.4 \pm 1.6, t=-0.5, p=0.632)$, ageadjusted $\mathrm{CCl}(1.1 \pm 1.9$ vs. $0.9 \pm 1.7, \mathrm{t}=-1.1, \mathrm{p}=0.253)$, NICE criteria $(58.4 \%$ vs. $58.6 \%$, $\mathrm{t}=-0.2, \mathrm{p}=0.982)$ or medication counts (excluding PD drugs) (3.7 \pm 3.1 vs. $3.1 \pm 2.8, \mathrm{t}=-$ 1.3, $\mathrm{p}=-$-182). No significant differences in comorbidities were seen between PD 
participants and controls, with the exception of cerebrovascular disease $(7.1 \%$ vs. $0 \%$, $\mathrm{p}<0.05)$.

Univariate linear regression analysis showed that more severe motor severity using the MDS-UPDRS-III score was associated with significantly higher GDS-15 scores $(p<0.001)$ and lower MoCA scores $(p<0.05)$. Both GDS-15 and MoCA scores were included in the basic model (table 1). The addition of individual multimorbidity measures did not significantly improve the model. 
Table 1 Predictors of MDS-UPDRS-III score

\begin{tabular}{|c|c|c|c|c|c|c|c|}
\hline & & O & r & 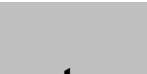 & & $95 \% \mathrm{C}$ & for $\beta$ \\
\hline & & $\beta$ & SE & $\mathbf{t}$ & $\mathbf{p}$ & Lower & Upper \\
\hline Basic & MoCA & -0.147 & 0.274 & -1.813 & 0.072 & -1.037 & 0.045 \\
\hline Modela $^{\mathbf{a}}$ & GDS-15 & 0.288 & 0.380 & 3.553 & 0.001 & 0.598 & 2.099 \\
\hline & $\begin{array}{l}\text { Basic Model }+ \\
\text { Disease Count }\end{array}$ & 0.014 & 0.573 & 0.161 & 0.872 & -1.040 & 1.225 \\
\hline & $\begin{array}{l}\text { Basic Model + } \\
\text { Medication Count } \\
\text { (Non-PD }^{\text {medications) }}{ }^{c}\end{array}$ & 0.038 & 0.332 & 0.446 & 0.656 & -0.509 & 0.806 \\
\hline & $\begin{array}{l}\text { Basic Model + } \\
\text { Medication Count } \\
\text { (All Prescribed }^{\text {medications) }}{ }^{d}\end{array}$ & 0.026 & 0.334 & 0.308 & 0.759 & -0.557 & 0.762 \\
\hline & $\begin{array}{l}\text { Basic Model + Age- } \\
\text { adjusted } \mathrm{CCl}\end{array}$ & -0.071 & 0.530 & -0.868 & 0.387 & -1.509 & 0.588 \\
\hline & $\begin{array}{l}\text { Basic Model + NICE } \\
\text { guideline-defined } \\
\text { multimorbidity }\end{array}$ & 0.003 & 2.082 & 0.035 & 0.972 & -4.046 & 4.191 \\
\hline a $\mathrm{R}=0.3$ & Adjusted $R^{2}=0.099, F=$ & $3.598, p$ & $=0.001$ & & & & \\
\hline${ }^{b} \mathrm{R}=0.33$ & $\Delta R^{2}=0.000, F=5.699$ & $=0.001$ & & & & & \\
\hline${ }^{\mathrm{c}} \mathrm{R}=0.33$ & $\Delta R^{2}=0.001, F=5.764, k$ & $=0.001$ & & & & & \\
\hline${ }^{\mathrm{d}} \mathrm{R}=0.33$ & $\Delta R^{2}=0.001, F=5.725$ & $=0.001$ & & & & & \\
\hline${ }^{e} \mathrm{R}=0.34$ & $\Delta R^{2}=0.005, F=5.972$ & $=0.001$ & & & & & \\
\hline${ }^{f} \mathrm{R}=0.33$ & $\Delta R^{2}=0.000, F=5.690, p$ & 0.001 & & & & & \\
\hline
\end{tabular}

Significant values indicated in bold.

Abbreviations: MDS-UPDRS-III = Movement Disorder Society Unified Parkinson's Disease Rating Scale III; MoCA = Montreal Cognitive Assessment; GDS-15 = Geriatric Depression Scale-15; $\mathrm{CCl}=$ Charlson Comorbidity Index .

Univariate analysis revealed that younger age, higher GDS-15 scores and higher MDS-UPDRS-III scores significantly predicted of poorer QoL (Table 2); these were included in the basic model, in addition to years of education which demonstrated a 
trend for prediction. Gender was also included in the basic model as gender difference in QoL have been previously reported. These accounted for $51.3 \%$ of the variance seen in PDQ-39 scores. The addition of all multimorbidity measures improved the predictive ability of the basic model except for NICE guideline-defined multimorbidity (Table 2, $p<0.05$ for all), demonstrating that multimorbidity was associated with lower QoL. The multimorbidity measures accounted for small changes in the models (1.52.5\%); medication count of all prescribed medications had the most influence on QoL $\left(\Delta R^{2}=0.026\right)$. 
Table 2 Predictors of PDQ-39 score

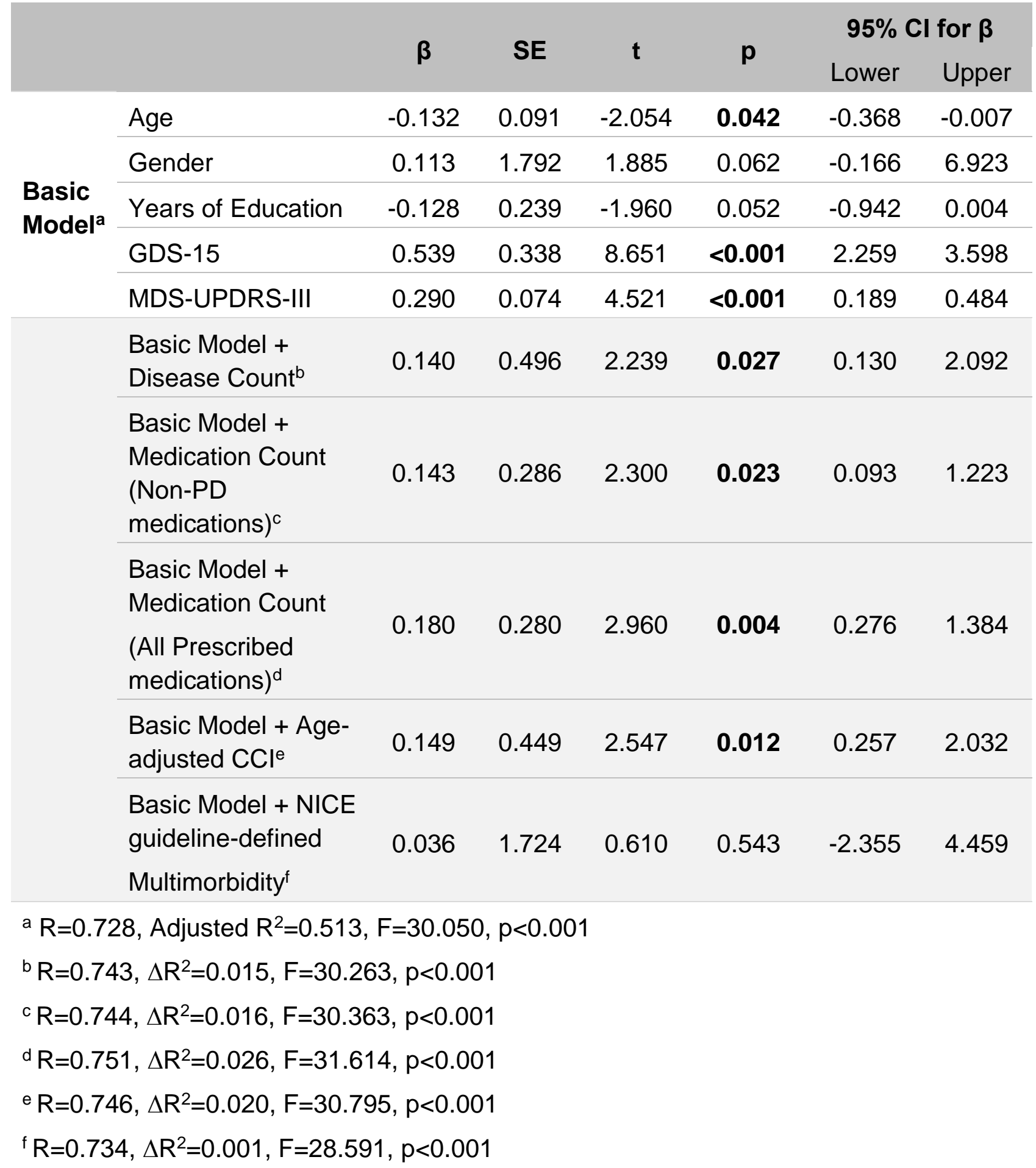

Significant values indicated in bold.

Abbreviations: PDQ-39 = Parkinson's Disease Questionnaire-39; GDS-15 = Geriatric Depression Scale-15; MDS-UPDRS-III = Movement Disorder Society Unified Parkinson's Disease Rating Scale III; CCI = Charlson Comorbidity Index. 


\section{Discussion}

Multimorbidity is an evolving concept, and to our knowledge, this is the first study to investigate the association between multimorbidity and the severity of PD in a large incident cohort, where we found a small but significant effect on QoL.

The burden of multimorbidity did not differ between PD participants and controls, except for when measured using a medication count of all prescribed medications, differing from previous findings [11].This may be accounted for by our use of the ICPC-2 conditions, which are more selective, minimising the overlap with NMS.

There is no universal tool for measuring PD-related multimorbidity. However, recent literature tends to use age-adjusted $\mathrm{CCl}$ as it is considered to be a robust, evidence-based method for the prediction of long-term QoL [12]. Medication counts are simple, however, given the multi-system nature of PD, the mean count of all prescribed medications was higher in patients with PD compared with controls. Non-PD medication count may improve accuracy in assessing multimorbidity in PD patients, eliminating PD-burden as a confounder.

We did not find an effect of multimorbidity on motor severity. This may relate to the fact that we were studying an incident cohort, with accordingly early disease (mean disease duration $=6.0$ months), reducing the heterogeneity of motor severity and the ability to detect the possible predictive powers of multimorbidity measures.

Leibson et al. [11] demonstrated a dose-response effect of comorbidities on worsening outcomes relating to mental disorders. Mitnitski et al. [13] provide a model of deficit accumulation, where the more pathophysiological processes an individual accumulates, the more vulnerable they become. Despite referring to mortality this may explain the relationship seen between multimorbidity and QoL. Multimorbidity may lead to physical or mental stress, resulting in a restricted capacity to function and lower QoL. A unified approach to care 
proposes a multifactorial theory for addressing patient outcomes, addressing the role of dynamic interactions between physiological functions. Outcomes are linked, and addressing individual outcomes, such as pathophysiological process, will in turn affect other patient outcomes in terms of morbidity and mortality exemplifying the importance of multimorbidity on QoL in early PD.

Clinically, multimorbidity is important because it is common and affects QoL Multimorbidity has an impact upon recruitment to clinical trials and carer strain. Thus, identifying it offers targets for medication rationalisation, multidisciplinary approach to care, and re-thinking of clinical trial design.

This study included a large well-defined cohort of participants with incident PD, and ageand sex-matched controls undergoing rigorous testing with validated research tools. Not all PD participants were dopamine naïve, therefore the extent of the relationship between multimorbidity and motor severity and QoL cannot be generalised to treatment-naïve patients. Patients who declined to participate tended to be older [9] and may be speculated to have greater comorbid disease counts and impairments.. Clinically, participants with PD would be expected to have more multimorbidity and worse general health; however, this was not reflected in the results.

\section{Conclusion}

Multimorbidity is associated with worse QoL in patients with early PD. Effective management of multimorbidity is important to achieve optimal QoL in this patient cohort, and implies that a multidisciplinary approach to treating PD should be instigated from diagnosis and not just in more advanced disease.

A longitudinal investigation of this cohort, focusing on the long-term impact of multimorbidity on motor severity, and QoL could clarify the influence of multimorbidity on disease 
progression. Furthermore, given the multi-system nature of PD, the development of a PDspecific multimorbidity measure, including the hybridisation of the medication count, non-PD medication count and age-adjusted $\mathrm{CCl}$, may improve specificity and predictive abilities of such a tool.

\section{Declaration of Sources of Funding}

This work was supported by the Incidence of Cognitive Impairment in Cohorts with Longitudinal Evaluation - Parkinson's Disease (ICICLE-PD) and was funded by Parkinson's UK [J-0802, G-1301, G-1507] and supported by the UK NIHR Biomedical Research Centre for Ageing and Age-related Disease award to the Newcastle upon Tyne Hospitals NHS Foundation Trust and Addenbrooke's NHS Trust. Ethical approval was obtained from Newcastle and North Tyneside 1 Research Ethics Committee.

\section{Conflict of interest}

None 


\section{Supplementary Data}

Demographics and characteristics of participants with PD and controls

\begin{tabular}{|c|c|c|c|c|c|c|}
\hline & \multicolumn{2}{|c|}{$\begin{array}{c}\text { PD } \\
(n=154)\end{array}$} & \multicolumn{2}{|c|}{$\begin{array}{l}\text { Controls } \\
(n=99)\end{array}$} & \multirow{2}{*}{$\begin{array}{c}\text { Test } \\
\text { statistic }\end{array}$} & \multirow[t]{2}{*}{$P$ value } \\
\hline & Mean & SD & Mean & SD & & \\
\hline Age (years) & 66.4 & 10.4 & 67.9 & 8.2 & $-1.3^{\dagger}$ & 0.214 \\
\hline $\begin{array}{c}\text { Gender (Male) } \\
\mathrm{n}(\%)\end{array}$ & $100(64.9)$ & - & $54(54.5)$ & - & $-1.6^{*}$ & 0.099 \\
\hline $\mathrm{MoCA}^{\ddagger}$ & 25.2 & 3.7 & 27.0 & 2.5 & -3.7 & $<0.001$ \\
\hline MMMSE & 28.6 & 1.3 & 29.0 & 1.2 & -2.7 & 0.006 \\
\hline GDS-15 & 2.8 & 2.5 & 1.0 & 1.5 & -6.9 & $<0.001$ \\
\hline BMI & 27.2 & 4.8 & 27.7 & 3.9 & -1.2 & 0.247 \\
\hline $\begin{array}{l}\text { Years of } \\
\text { Education }\end{array}$ & 12.8 & 3.8 & 13.1 & 3.4 & -1.2 & 0.238 \\
\hline NART & 114.7 & 10.7 & 115.9 & 8.7 & -0.5 & 0.635 \\
\hline $\begin{array}{l}\text { Duration of PD } \\
\text { (Months) }\end{array}$ & 6.0 & 4.6 & - & - & - & - \\
\hline MDS-UPDRS-II & 10.9 & 5.8 & - & - & - & - \\
\hline MDS-UPDRS-III & 26.9 & 12.1 & - & - & - & - \\
\hline \multicolumn{7}{|l|}{ H\&Y Scale, $n(\%)$} \\
\hline 1 & $35(22.7)$ & & & & & \\
\hline 2 & $88(57.1)$ & - & - & - & - & - \\
\hline 3 & $30(19.5)$ & & & & & \\
\hline 4 & $1(0.6)$ & & & & & \\
\hline PDQ-39 & 18.7 & 14.4 & - & - & - & - \\
\hline LEDD (mg/day) & 178.0 & 148.2 & - & - & - & - \\
\hline $\begin{array}{c}\mathrm{MCl} \text { present } \\
\mathrm{n}(\%)\end{array}$ & 65 (42.2) & - & $21(21.2)$ & 0.4 & $-3.4^{*}$ & 0.001 \\
\hline
\end{tabular}

Abbreviations: MoCA = Montreal Cognitive Assessment; MMMSE = Mini-Mental State Examination; GDS15 = Geriatric Depression Scale-15; BMI = Body Mass Index; NART = National Adult Reading Test; MDSUPDRS = Movement Disorder Society Unified Parkinson's Disease Rating Scale; H\&Y = Hoehn and Yahr Scale; PDQ-39 = Parkinson's Disease Questionnaire; LEDD = Levodopa Equivalent Daily Dose; $\mathrm{MCI}=$ Mild Cognitive Impairment using 1.5SD cut off.

All data are mean and standard deviations except where indicated. All data are non-parametric and test statistics are $z$ scores from Mann-Whitney U-test except where indicated. ${ }^{*}$ Chi squared test; †Independent t-test. Significant values indicated in bold.

$\ddagger \mathrm{n}=15$ did not complete MoCA. 


\section{References}

1. Csoti I., Jost W.H., and Reichmann H. (2016). Parkinson's disease between internal medicine and neurology. J Neural Transm (Vienna). 123(1): p. 3-17. DOI:https://doi.org/10.1007/s00702-015-1443-z

2. Yarnall A.J., Sayer A.A., Clegg A., Rockwood K., Parker S., Hindle J.V. (2017). New horizons in multimorbidity in older adults. Age Ageing. 46(6): p. 882-888. DOI:https://doi.org/10.1093/ageing/afx150

3. Fortin M., Stewart M., Poitras M.E., Almirall J., Maddocks H. (2012) A systematic review of prevalence studies on multimorbidity: toward a more uniform methodology. Ann Fam Med. 10(2): p. 142-51. DOI:https://doi.org/10.1370/afm.1337

4. National Institue for Health and Care Excellence (NICE). (2016). Multimorbidity: clinical assessment and management. NICE: London.

5. Ahmadi B., Alimohammadian M., Yaseri M., Majidi A., Boreiri M., Islami F., et al. (2016). Multimorbidity: Epidemiology and Risk Factors in the Golestan Cohort Study, Iran: A Cross-Sectional Analysis. Medicine. 95(7): p. e2756. DOI:https://doi.org/10.1097/MD.0000000000002756

6. Calderón-Larrañaga A., Poblador-Plou B., González-Rubio F., Gimeno-Feliu L.A., Abad-Díez J.M, and Prados-Torres A. (2012). Multimorbidity, polypharmacy, referrals, and adverse drug events: are we doing things well? The British Journal of $\begin{array}{llll}\text { General } & \text { Practice. } & \text { 62(605): } & \text { e821-e826. }\end{array}$ DOI:https://doi.org/10.3399/bjgp12X659295

7. Hassan A., Wu S.S., Schmidt P., Dai Y., Simuni T., Giladi N., et al. (2013). High rates and the risk factors for emergency room visits and hospitalization in Parkinson's disease. Parkinsonism \& Related Disorders. 19(11): p. 949-954. DOI:https://doi.org/10.1016/j.parkreldis.2013.06.006 
8. McLean G., Hindle J.V., Guthrie B., Mercer S.W. (2017). Co-morbidity and polypharmacy in Parkinson's disease: insights from a large Scottish primary care database. BMC Neurology. 17: p. 126. DOl:https://doi.org/10.1186/s12883-0170904-4

9. Yarnall A.J., Breen D.P., Duncan G.W., Khoo T.K., Coleman S.Y., Firbank M.J., et al. (2014). Characterizing mild cognitive impairment in incident Parkinson disease - The $\begin{array}{llll}\text { ICICLE-PD } & \text { study. } & \text { 82(4). }\end{array}$ DOI:https://doi.org/10.1212/WNL.0000000000000066

10. Tomlinson C.L., Stowe R., Patel S., Rick C., Gray R. Clarke C.E. (2010) Systematic review of levodopa dose equivalency reporting in Parkinson's disease. Mov Disord. 25(15): p.2649-53. DOI:https://10.1002/mds.23429

11. Leibson C.L., Maraganore D.M., Bower J.H., Ransom J.E., O'Brien P.C., Rocca W.A. (2006). Comorbid conditions associated with Parkinson's disease: a populationbased study. Mov Disord. 21(4): p. 446-55. DOI:https://doi.org/10.1002/mds.20685

12. Huntley A.L., Johnson R., Purdy S., Valedras J.M., Salisbury C. (2012). Measures of Multimorbidity and Morbidity Burden for Use in Primary Care and Community Settings: A Systematic Review and Guide. Annals of Family Medicine. 10(2): p. 134141. DOI:https://doi.org/10.1370/afm.1363.

13. Mitnitski A., Bao L., Rockwood K. (2006). Going from bad to worse: a stochastic model of transitions in deficit accumulation, in relation to mortality. Mech Ageing Dev. 127(5): p. 490-3. DOI:https://doi.org/10.1016/j.mad.2006.01.007 